\title{
Efficient Proxy Re-encryption with Private Searching in the Untrusted Cloud
}

\author{
${ }^{\text {a } 1}$ Xi Chen, ${ }^{\text {a }}$ Yong Li \\ ${ }^{a}$ Key Laboratory of Communication \& Information Systems (Beijing Jiaotong University), Beijing Municipal \\ Commission of Education, Beijing 100044, China
}

\begin{abstract}
As promising as cloud computing is, this paradigm brings forth new security and privacy challenges when operating in the untrusted cloud scenarios. In this paper, we propose a new cryptographic primitive Proxy Reencryption with Private Searching (PRPS for short). The PRPS scheme enables the data users and owners efficiently query and access files storaged in untrusted cloud, while keeping query privacy and data privacy from the cloud providers. The concrete construction is based on proxy re-encryption, public key encryption with keyword search and the dual receiver cryptosystem. The scheme is semantically secure under the BDH assumption.
\end{abstract}

Index Terms: public key encryption with keyword search; proxy re-encryption; untrusted cloud; private searching

(C) 2012 Published by MECS Publisher. Selection and/or peer review under responsibility of the Research Association of Modern Education and Computer Science

\section{Introduction}

Cloud computing is an important trend which is beginning to fulfill the early promise of the Internet and creating unanticipated change in computing paradigm. However, a significant barrier to the adoption of cloud computing is that data owners fear of confidential data leakage and lose of privacy in the cloud [1]. These concerns originate from the fact that cloud providers are usually operated by commercial providers which are very likely to be outside of the trusted domain of the data owners or users. Data confidentiality against cloud providers is hence frequently desired when data owners outsource data for storage in the cloud [2].

Our work is motivated by the following scenario. Data owners, cloud storage providers and data users are separated geographically. Data owner stores his files in an encrypted form in the untrusted cloud, and retrieves them wherever and whenever he wants. The user sends a query for files containning certain keywords to the cloud provider. The desired requirements are: 1) The user can decrypt the files uploaded by the data owner with his private key; 2) The cloud provider can search whether the encrypted files contain some keywords; 3) The 
cloud provider oughts to keep blind to the files content and the query keywords of the user; 4) The user could finish query and decryption with a thin client which demands computing overhead as small as possible.

\subsection{Related work}

Proxy Re-Encryption (PRE). PRE is a cryptographic primitive, where a (potentially untrusted) proxy is given a re-encryption key $r k_{1 \rightarrow 2}$ that allows it to translate a message $m$ encrypted under public key $p k_{1}$ into a ciphertexts under a public key $p k_{2}$, without being able to see anything about the encrypted messages. In [3], Ateniese $e t$ al. proposed a single-use, unidirectional, but not transparent Proxy Re-Encryption schemes based on bilinear maps.

Public key encryption with keyword search (PEKS). In PEKS scheme, Alice creats a trapdoor with her private key and a keyword, and sends it to S. S uses a test algorithm with inputing encrypted keyword, trapdoor and user's public key. If matches, it outputs 1 and 0 otherwise. PEKS supports that a user could search for some files containning certain keywords in untrusted storage servers, and at the same time, the servers keep blind to the privacy of file and the keyword. In [4], Boneh et al. proposed a public key encryption with keyword search scheme.

Dual receiver cryptosystem. Diament et al. [5] first introduced the notion of an effcient dual receiver cryptosystem, which enables a ciphertext to be decrypted by two independent receivers. The main disadvantage of the dual receiver cryptosystem is that the server needs to send an auxiliary private key to a client for decrypting a partial ciphertext, which is insecure in the real environment [6].

Liu et al. [6] improved the PEKS by inspiring the idea of dual receiver cryptosystem, and proposed an effcient privacy preserving keyword search scheme. However, this scheme is one specific case applicable in the setting that the data owner and data user is the same one. Shao et al. [7] introduced the concept of proxy re-encryption with keyword search (PRES), in particular the concept of bidirectional PRES, against the chosen ciphertext attack. Note that the third party is trusted, and this scheme improved the security level with the sacrifice of efficiency.

\subsection{Our contributions}

We proposed a new cryptographic primitive, Proxy Re-encryption with Private Searching (PRPS), and the new PRPS construction combines technologies from PRE, PEKS and dual receiver cryptosystem. The PRPS scheme is able to protect the data privacy and the users' queries privacy simultaneously during the search process. And it is provably secure under the BDH assumption in random oracle model. In addition, the PRPS scheme enables the decrease of computing overhead for the user and reduces the modification of encrypted storage when different users accessing the cloud provider.

\section{Preliminaries}

Let $G_{1}$ and $G_{2}$ be two cyclic groups of some large prime order $q$. We view $G_{1}$ as an additive group and $G_{2}$ as a multiplicative group.

Definition 2.1 (Bilinear Maps): A bilinear map $e: G_{1} \square G_{1} \square G_{2}$ is a map with the following properties: (1) Bilinearity: for any integers $x, y \square[1, q]$, we have $e\left(g^{x}, g^{y}\right) \square e(g, g)^{x y}$. (2) Computability: given $g, h \square G_{1}$, there is a polynomial time algorithms to compute $e(g, h) \square G_{2}$. (3) Non-degeneracy: if $g$ is a generator of $G_{1}$, then $e(g, g)$ is a generator of $G_{2}$.

Definition 2.2 (BDH Problem): Given a random element $g \square G_{1}$, as well as $g^{x}, g^{y}$ and $g^{z}$, for some $x, y, z \square Z_{q}^{*}$, compute $e(g, g)^{x y z} \square G_{2}$. 


\section{Proxy Re-encryption with Private Searching}

Definition 3.1 Proxy Re-encryption with Private Searching (PRPS) scheme consists of seven randomized polynomial time algorithms as follows:

- Key Generation (KG): takes a suffciently large security parameter $K_{1}$ as input, and produces a public/private key pair $\left(A_{p u b}, A_{p r i v}\right)$ for a data owner $A$. We write $K G\left(K_{1}\right) \square\left(A_{p u b}, A_{p r i v}\right)$. Let $K_{2}$ be a suffciently large security parameter, we write $K G\left(K_{2}\right) \square\left(S_{p u b}, S_{p r i v}\right)$ for the cloud provider $S$, where $S_{p u b}, S_{p r i v}$ are public /private key respectively. Let $K_{3}$ be a suffciently large security parameter, we write $K G\left(K_{3}\right) \square\left(U_{p u b}, U_{p r i v}\right)$ for the data user $U$, where $U_{p u b}, U_{p r i v}$ are public/private key respectively.

- Encryption (E): this algorithm is performed by data owner $A$ to encrypt the keyword $W_{i}\left(i \square Z^{+}\right.$) and message $m$. Correspondingly, two parts, KWEnc and EMBEnc constitutes Encryption.

1) KWEnc: is a public key encryption algorithm that takes a public key $A_{p u b}$ and a key word $W_{i}\left(i \square Z^{+}\right)$as inputs, and produces $W_{i}^{\prime} s$ ciphertext $C_{W_{i}} \square C_{w}$. We write $\operatorname{KWEnc}\left(A_{p u b}, W_{i}\right) \square \mathrm{C}_{W_{i}}$

2) EMBEnc: is a public key encryption algorithm that takes public keys $S_{p u b}, A_{p u b}$ and message $m \square M$ as inputs, and produces $m$ 's ciphertext $C_{m}$. We write $\operatorname{EMBEnc}\left(S_{p u b}, A_{p u b}, m\right) \square C_{m}$.

- Re-Encryption Key Generation (RG): A data owner takes private key $A_{p r i v}$ and user's public key $U_{p u b}$ as inputs, and produces the re-encryption key $r k_{A \rightarrow U}$. We write $R G\left(A_{p r i v}, U_{p u b}\right) \square r k_{A \rightarrow U}$.

- TCompute: User takes private key $U_{\text {priv }}$ and a keyword $W_{j}\left(j \square Z^{+}\right)$as inputs, and produces $W_{j}^{\prime} s$ trapdoor $T_{W_{j}}$. We write $\operatorname{TCompute}\left(U_{\text {priv }}, W_{j}\right) \square T_{W_{j}}$.

- Re-Encryption (R): The cloud provider takes re-encryption key $r k_{A \rightarrow U}$, ciphertext $C_{m}$ and some intermediate result $\square$ as the inputs, and produces ciphertext $C_{m}^{\prime} s$ re-encrypted ciphertext $C_{U}$. We write $\mathrm{Re} \square \operatorname{Encryption}\left(\square, r k_{A \rightarrow U}, C_{m}\right) \square C_{U}$.

- Test: The cloud provider takes re-encryption key $r k_{A \rightarrow U}$, an encrypted keyword $C_{W_{i}}$ and a trapdoor $T_{W_{j}}$ as inputs, and produces " 1 " if $W_{i} \square W_{j}$ or " 0 " otherwise. This algorithm is to check whether the ciphertext $C_{W_{i}}$ matches the trapdoor $T_{W_{j}}$.

- Decryption (D): The user takes private key $U_{\text {priv }}$ and re-encrypted ciphertext $C_{U}$ as inputs, and outputs the plaintext $m$.

Definition 3.2 (Semantic Security of KWEnc): Given a public key encryption algorithm KWEnc which encrypts keywords using $A_{p u b}$, let $\square_{1}$ be a polynomial time IND-CPA adversary that can adaptively ask for the trapdoor $T_{W_{i}}$ for any keyword $W_{i} \square W$ of its choice. $\square_{1}$ first chooses two keywords $W_{0}$ and $W_{1}$, which are not to be asked for trapdoors previously, and sends them to KWEnc. And then KWEnc picks a random element $b_{1} \square\{0,1\}$ and gives $\square_{1}$ the ciphertext $C_{W_{b_{1}}} \square \operatorname{KWEnc}\left(A_{p u b}, W_{b_{1}}\right)$. Finally, $\square_{1}$ outputs a guess $b_{1}^{\prime} \square\{0,1\}$ for $b_{1}$. We define the advantage of $\square_{1}$ in breaking KWEnc as $A d v_{\mathrm{Al}_{1}}(k) \square\left|\operatorname{Pr}\left[b_{1} \square b_{1}^{\prime}\right] \square \frac{1}{2}\right|$. KWEnc is semantically secure if for any polynomial time adversary $\square_{1}, A d v_{\hat{A}_{1}}(k)$ is negligible.

Definition 3.3 (Semantic Security of EMBEnc): Given a public key encryption algorithm EMBEnc which encrypts the message using $A_{p u b}$ and $S_{p u b}$. Let $\square_{2}$ be a polynomial time IND-CPA adversary that can adaptively ask for the ciphertext for any message $m_{i} \square M$ of its choice. We use subscript $T$ to denote the target user, $x$ to denote the adversarial users, and $h$ to denote the honest users (other than $T$ ). The input marked with a '*' is optional. $\square_{2}$ first chooses two messages $m_{0}$ and $m_{1}$, which are not to be asked for the ciphertext previously, and 
sends them to EMBEnc. And then EMBEnc picks a random $b_{2}^{\prime} \square\{0,1\}$ and gives $\square_{2}$ the ciphertext $C_{m_{b_{2}}} \square \operatorname{EMBEnc}\left(A_{p u b}, S_{p u b}, m_{b_{2}}\right)$. Finally, $\square_{2}$ outputs a guess $b_{2}^{\prime} \square\{0,1\}$ for $b_{2}$. That is, for all PPT algorithms $A_{k}$,

$$
\begin{array}{r}
\operatorname{Pr}\left[\left(p k_{T}, s k_{T}\right) \square K G\left(1^{k}\right),\left\{\left(p k_{x}, s k_{x}\right) \square K G\left(1^{k}\right)\right\},\right. \\
\left\{r k_{x \rightarrow T} \square R G\left(p k_{x}, s k_{x}, p k_{T}, s k_{T}^{*}\right)\right\}, \\
\left\{\left(p k_{h}, s k_{h}\right) \square K G\left(1^{k}\right)\right\}, \\
\left\{r k_{T \rightarrow h} \square R G\left(p k_{T}, s k_{T}, p k_{h}, s k_{h}^{*}\right)\right\}, \\
\left\{r k_{h \rightarrow T} \square R G\left(p k_{h}, s k_{h}, p k_{T}, s k_{T}^{*}\right)\right\}, \\
\left(m_{0}, m_{1}, \mapsto\right) \square A_{k}\left(p k_{T},\left\{\left(p k_{x}, s k_{x}\right)\right\},\left\{p k_{h}\right\},\left\{r k_{x \rightarrow T}\right\},\left\{r k_{T \rightarrow h}\right\},\left\{r k_{h \rightarrow T}\right\}\right), \\
b_{2} \square\{0,1\}, b_{2}^{\prime} \square \quad A_{k}\left(\mapsto, E M B E n c\left(p k_{T}, m_{b_{2}}\right)\right): \\
\left.b_{2} \square b_{2}^{\prime}\right] \triangleleft 1 / 2 \square 1 / p o l y(k)
\end{array}
$$

We define the advantage of $\square_{2}$ in breaking EMBEnc as $A d v_{\mathrm{A}_{2}}(k) \square\left|\operatorname{Pr}\left[b_{2} \square b_{2}\right] \square \frac{1}{2}\right|$.We say that EMBEnc is semantically secure if for any polynomial time adversary $\square_{2}, A d v_{\mathbf{A}_{2}}(k)$ is negligible.

Definition 3.4 (Semantic Security of PRPS): Given an PRPS scheme consisting of KWEnc and EMBEnc, it takes a security parameter $K$ as input and runs the key generation algorithm $\mathrm{KG}$ to generate the public/private key pairs $\left(A_{p u b}, A_{p r i v}\right),\left(S_{p u b}, S_{p r i v}\right)$ and $\left(U_{p u b}, U_{p r i v}\right)$. Given an adversary $\square$ consisting of two polynomial time algorithms $\square_{1}$ and $\square_{2}, \square_{1}$ initiates attacks on KWEnc and $\square_{2}$ initiates attacks on EMBEnc. We say that the PRPS Scheme is semantically secure if for any adversary $\square, A d v_{\mathrm{A}_{\mathrm{A}}}(k) \square A d v_{\mathrm{A}_{1}}(k) \square A d v_{\mathrm{A}_{2}}(k)$ is negligible.

\section{Construction for PRPS}

We assume that the scheme is composed of the following entities, the data owner, data users, and cloud providers. To access data files shared by the data owner, data users download data files of their interest from cloud providers and then decrypt. The users are assumed to have the only access privilege of data file reading. The cloud providers are assumed to have abundant storage capacity and computation power.

In our scheme, cloud providers are viewed as "honest but curious", which means they follow the proposed protocol in general, but try to find out as much secret information as possible. Cloud providers might collude with malicious users for the purpose of harvesting file contents when it is highly beneficial. Communication channel between the data owner/users and cloud providers are assumed to be secured. Users may work independently or cooperatively.

The main design goal is to help the data users achieve efficient private querying and downloading the encryted files stored in cloud providers. The data owner won't need to re-encryt the files in cloud provider for different users. We also want to prevent cloud providers from being able to learn both the data file contents and user queries information.

Suppose data owner $A$ is about to store an encrypted file with keywords $W_{1}, \ldots W_{l}$ on a cloud storage $S$, where $l \square Z^{+}$. Keywords may be words in headline or stored date, and are relatively small. $A$ encrypts the file message using his public key $A_{p u b}$, the cloud storage's public key $S_{p u b}$. And then $A$ encrypts keywords $W_{1}, \ldots W_{l}$ using his public key $A_{p u b}$. The file deposited in the cloud storage $S$ by the data owner $A$ is as follows:

$$
M S G_{U 2 S} \square\left[\operatorname{EMBEnc}\left(A_{p u b}, S_{p u b}, m\right), K W E n c\left(A_{p u b}, W_{1}\right), \ldots, \operatorname{KWEnc}\left(A_{p u b}, W_{l}\right)\right]
$$

where EMBEnc , KWEnc are public key encryption algorithms. Finally, $A$ appends to the encrypted file message with all the encrypted keywords and sends $M S G_{U 2 S}$ to $S$. 
Given a suffciently large security parameter $K \square Z^{+}$, two groups $G_{1}$ and $G_{2}$ of prime order $q$, and a bilinear map $e: G_{1} \square G_{1} \square G_{2}, g, h \square G_{1}, Z \square e(g, g) \square G_{2}$, where $g$ is a generator of $G_{1}$. Then it chooses two hash functions

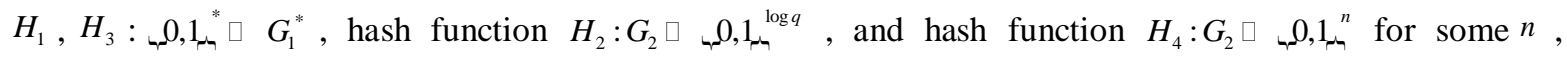
where $H_{1}, H_{2}, H_{3}$ and $H_{4}$ are random oracles. Finally, it picks three random elements $a, b, c \square Z_{q}^{*}$ and computes $g^{a}, g^{b}$ and $g^{c}$.The plaintext space includes $M \square\{0,1\}^{n}$ and $W \square\{0,1\}^{*}$. The ciphertext space includes $C_{M} \square G_{1}^{*} \square, 0,1_{\llcorner}^{n}$ and $C_{W} \square G_{2}$.

- Key Generation (KG): The data owner $A$ 's public key is $A_{p u b} \square g^{a}$ with the corresponding private key $A_{\text {priv }} \square a$; the user $U$ 's public/private key is $U_{p u b} \square g^{b}, U_{p r i v} \square b$ respectively. The cloud provider $S$ 's public key is $S_{p u b} \square g^{c}$ with the corresponding private key $S_{p r i v} \square c$.

- Encryption (E): This encryption algorithm consists of KWEnc and EMBEnc. The data owner first picks a random element $r \square Z_{q}^{*}$.

1) KWEnc $\left(E_{1}\right)$ : To encrypt $m$ 's keywords $W_{1}, \ldots, W_{k}\left(k \square Z^{+}\right)$under a data owner's public key $g^{a}$ and a random element $r$, it computes $H_{2}\left(e\left(g^{a}, H_{1}\left(W_{i}\right)\right)^{r}\right)$, where $W_{i} \square W_{1}, \ldots, W_{k m}$, sets the ciphertext $C_{W_{i}} \square H_{2}\left(e\left(g^{a}, H_{1}\left(W_{i}\right)\right)^{r}\right)$.

2) EMBEnc $\left(E_{2}\right)$ : To encrypt the file message $m$ under data owner's public key $g^{a}$, cloud provider's public key $g^{c}$ and random element $r$, it picks a random element $\neg \square \downarrow_{\downarrow} 0,1_{\sim \sim}^{n}$, and computes $u_{1} \square h^{r}$, $u_{2} \square \rightarrow \square H_{4}\left(e\left(h^{a}, g^{c}\right)^{r}\right), u_{3} \square m \llbracket e\left(H_{3}(\rightarrow), g^{a}\right)^{r}$, and sets the ciphertext $C_{m} \square\left(u_{1}, u_{2}, u_{3}\right)$.

- Re-Encryption KeyGeneration (RG): Data owner $A$ delegates to user $U$ by publishing the re-encryption key $r k_{A \rightarrow U} \square g^{a b r}$, computed with $U$ 's public key $g^{b}$.

- Tcompute: To retrieve the file containing keyword $W_{j}\left(j \square Z^{+}\right)$, user computes the trapdoor $T_{W_{j}} \square H_{1}\left(W_{j}\right)^{1 / b}$ using his/her private key $U_{p r i v} \square b$, then sends the trapdoor to the cloud provider.

- Re-Encryption $(R)$ : to change the ciphertext $C_{m} \square\left(u_{1}, u_{2}, u_{3}\right)$ for $A$ into a ciphertext $C_{U} \square\left(u_{3}, u_{4}\right)$ for $U$ under the re-encyption key $r k_{A \rightarrow U} \square g^{a b r}$, it computes $u_{4} \square e\left(H_{3}(\rightarrow), r k_{A \rightarrow U}\right) \square e\left(H_{3}(\rightarrow), g^{a b r}\right)$. The cloud provider sends $C_{U}$ to the user.

Note. Since $-\square u_{2} \square H_{4}\left(e\left(h^{a}, g^{c}\right)^{r}\right) \square u_{2} \square H_{4}\left(e\left(g^{a}, h^{r}\right)^{c}\right)$, the cloud provider can compute the intermediate value $\rightarrow$ with its private key $c$.

- Test: To determine whether a given file contains keyword $W_{j}$, the cloud provider tests whether $C_{W_{i}} \square H_{2}\left(e\left(r k_{A \rightarrow U}, T_{W_{j}}\right)\right)$. If so, Test $\left(r k_{A \rightarrow U}, C_{W_{i}}, T_{W_{j}}\right)$ outputs 1 , and 0 otherwise.

Note. If $W_{i} \square W_{j}$, since $C_{W_{i}} \square H_{2}\left(e\left(g^{a}, H_{1}\left(W_{i}\right)\right)^{r}\right)$, then

$C_{W_{i}} \square H_{2}\left(e\left(g^{a}, H_{1}\left(W_{j}\right)\right)^{r}\right) \square H_{2}\left(e\left(g^{a b r}, H_{1}\left(W_{j}\right)^{1 / b}\right)\right) \square H_{2}\left(e\left(r k_{A \rightarrow U}, T_{W_{j}}\right)\right)$

- Decryption (D) : Given the ciphertext $C_{U} \square\left(u_{3}, u_{4}\right)$, it computes $m \square u_{3} /\left(u_{4}\right)^{\frac{1}{U_{\text {priv }}}} \square u_{3} /\left(u_{4}\right)^{\frac{1}{b}}$ to recover the message $m$.

Note that: $\frac{u_{3}}{\left(u_{4}\right)^{\frac{1}{b}}} \square \frac{m \llbracket e\left(H_{3}(-), g^{a}\right)^{r}}{\left(e\left(H_{3}(-), g^{a b}\right)^{r}\right)^{\frac{1}{b}}} \square \frac{m \llbracket e\left(H_{3}(-), g^{a}\right)^{r}}{e\left(H_{3}(-), g^{a}\right)^{r}} \square m$. 


\section{Security Analysis}

Lemma 5.1 (Privacy for Keyword) Let $H_{1}$ be a random oracle from $\{0,1\}^{*}$ to $G_{1}^{*}$ and $H_{2}$ be a random oracle from $G_{2}$ to $\{0,1\}^{\log q}$. Suppose $\square_{1}$ be an IND-CPA adversary that has the advantage $\square_{1}$ in breaking KWEnc. Suppose $\square_{1}$ makes at most $q_{H_{2}} \triangleright 0$ hash queries to $H_{2}$ and at most $q_{T} \triangleright 0$ trapdoor queries. Then there is an algorithm $B_{1}$ that solves the BDH problem with the advantage at least $\square_{1} \square 2 \square_{1} /\left\{e \square q_{H_{2}} \square\left(1 \square q_{T}\right)\right\}$, and a running time $O\left(\right.$ time $\left.\left(\square_{1}\right)\right)$.

Lemma 5.2 (Privacy for Message) Let $H_{3}$ be a random oracle from $\{0,1\}^{*}$ to $G_{1}^{*}$ and $H_{4}$ be a random oracle from $G_{2}$ to $\{0,1\}^{n}$. Let $\square_{2}$ be an IND-CPA adversary that has the advantage $\square_{2}$ against EMBEnc. Suppose $\square_{2}$ makes $q_{H_{3}} \triangleright 0$ hash function queries to $H_{3}$ and $q_{R} \triangleright 0$ queries to Request. Then there is an algorithm $B_{2}$ that solves the BDH problem with the advantage at least $\square_{2}^{\prime} \square 2 \square_{2} / q_{H_{3}} q_{R}$ and a running time $O\left(\operatorname{time}\left(\square_{2}\right)\right)$.

Theorem 5.1 (Security for PRPS) Suppose the hash functions $H_{1}, H_{2}, H_{3}$ and $H_{4}$ are random oracles. Let $\square$ be an IND-CPA adversary consisting of two polynomial time algorithms $\square_{1}$ and $\square_{2}$. Let $\square_{1}$ be an INDCPA adversary that has the advantage $\square_{1}$ in breaking KWEnc. Suppose $\square_{1}$ makes $q_{T} \triangleright 0$ trapdoor queries and $q_{\mathrm{H}_{2}} \triangleright 0$ hash queries to $H_{2}$. Let $\square_{2}$ be an IND-CPA adversary that has the advantage $\square_{2}$ against EMBEnc. Suppose $\square_{2}$ makes $q_{H_{3}} \triangleright 0$ hash function queries to $H_{3}$ and $q_{R} \triangleright 0$ queries to Request. Let $\square$ be an IND-CPA adversary that has the advantage $\square \square \square_{1} \square \square_{2}$ against the PRPS scheme. Then there is an algorithm $\square$ that solves the BDH problem with the advantage at least:

$$
A d v_{\mathrm{B}} \square 2 \square_{1} /\left\{e \square q_{H_{2}} \square\left(1 \square q_{T}\right)\right\} \square 2 \sqsupset_{2} / q_{H_{3}} q_{R}
$$

Here $e \square 2.71$ is the base of the natural logarithm. The running time of $\square$ is $O(\operatorname{time}(\square))$.

Due to page limitation, the details of formal security proof and some remarks are provided in the full version.

\section{Conclusions}

In this paper, we propose an efficient proxy re-encryption with private searching (PRPS) scheme in the untrusted cloud. We exploit proxy re-encryption and uniquely combining it with techniques of public key encryption with keyword search and dual receiver cryptosystem. PRPS allows users and data owners to query and access files storaged in untrusted cloud provider, while maintainning query privacy and data privacy. It allows user to decrypt the files efficiently. The PRPS scheme is proven semantically secure in the random oracle model.

\section{Acknowledgements}

This work is partially supported by National High Technology Research and Development Program of China (863 Program) under Grant No. 2009AA01Z423 and the Fundamental Research Funds for the Central Universities under Grant No. 2009JBM004.

\section{References}

[1] M. Armbrust, A. Fox, R. Griffith, A. D. Joseph, R. H. Katz, A. Konwinski, G. Lee, D. A. Patterson, A. Rabkin, I. Stoica, and M. Zaharia, “Above the clouds: A berkeley view of cloud computing," University of California, Berkeley, Tech. Rep. USB-EECS-2009-28, Feb 2009.

[2] Shucheng Yu, Cong Wang, Kui Ren and Wenjing Lou, "Achieving Secure, Scalable, and Fine-grained Data Access Control in Cloud Computing," Proceedings of INFOCOM 2010. IEEE,2010.

[3] G. Ateniese, K. Fu, M. Green, S. Hohenberger, "Improved proxy re-encryption schemes with applications to secure distributed storage," ACM Transactions on Information and System Security (TISSEC). 9 (1) (2006) 1-30. 
[4] D. Boneh, G. Crescenzo, R. Ostrovsky, and G. Persiano, "Public Key Encryption with Keyword Search," Proceedings of Eurocrypt 2004, LNCS 3027, Springer-Verlag. 2004. pp. 506-522.

[5] T. Diament, H. K. Lee, A. D. Keromytis and M. Yung, "The Dual Receiver Cryptosystem and its Application," Proceedings of the ACM CCS 2004, pp. 330-343.

[6] Qin Liu, Guojun Wang, Jie Wu, “An Efficient Privacy Preserving Keyword Search Scheme in Cloud Computing," CSE'09,vol.2, 2009 , pp. 715 - 720.

[7] J. Shao, Z. Cao, X. Liang, H. Lin, "Proxy re-encryption with keyword search," Inf. Sci. 180 (2010) 25762587. 